\title{
BUNCH LENGTHENING AND ENERGY SPREAD INCREASING IN ELECTRON STORAGE RINGS
}

\author{
J. Gao, Laboratoire de L'Accélérateur Linéaire, IN2P3-CNRS \\ et Université de Paris-Sud, 91405 Orsay cedex, France
}

\section{Abstract}

A theory of bunch lengthening and energy spread increasing in electron storage rings is established by introducing the concept of collective random excitations. Analytical formulae are established to calculate the equilibrium bunch length and the energy spread in the whole current range. Some experimental results are compared with those predicted by the theory and the comparison results are quite well.

\section{INTRODUCTION}

The phenomenon of bunch lengthening as a single bunch effect in an electron storage ring was first observed in ACO [1] at Orsay and later in other machines.

The first empirical formula [1] found in ACO to describe the variation of the bunch duration $\left(\sigma_{\tau}\right)$ with respect to the average bunch current $\left(I_{b}\right)$ and the beam energy $(E)$, written

$$
\sigma_{\tau}^{2}(\mathrm{~ns})=\sigma_{\tau 0}^{2}(\mathrm{~ns})\left(1+2 \times 10^{-3} \frac{I_{b}(\mathrm{~mA})}{E^{4}(\mathrm{GeV}) \sigma_{\tau}(\mathrm{ns})}\right)
$$

had a great impact on the potential well distortion (PWD) theory of longitudinal instabilities in electron storage rings established afterwards [2] [3]. The theories largely used to describe the bunch lengthening phenomenon are potential well distortion and microwave instability [4] theories. However, as stated by Chao [5] and Gareyte [6] the field of bunch lengthening is still an open area to more detailed investigations and analytical explanations.

In this paper, we try to give a theory which describes the variations of the bunch length and the energy spread with respect to the bunch current covering the whole current range.

\section{NATURAL BUNCH LENGTH AND ENERGY SPREAD}

It is well known that in an electron storage ring [7] an electron will lose its energy due to synchrotron radiations. Since this radiation is compensated by the rf cavities in the ring there exists a damping effect on the synchrotron oscillation with the corresponding damping time $\tau_{\varepsilon}=2 T_{0} / \frac{d \mathcal{U}_{0}}{d E}$, where $T_{0}$ is the revolution period, $\mathcal{U}_{0}$ is the synchrotron energy lost per electron per turn, and $E$ is the electron energy. In reality, the synchrotron radiation energy loss is in the form of randomly emitted photons, and this random quantum excitations together with the previously mentioned synchrotron radiation damping effects result in the single particle equilibrium energy spread, $\sigma_{\varepsilon_{0}}$, and single particle "bunch length", $\sigma_{z_{0}}$, expressed as

$$
\sigma_{\varepsilon_{0}}^{2}=\frac{\tau_{\varepsilon}}{4}<\mathcal{N}_{p}<u_{p}^{2}>>
$$

and

$$
\sigma_{z_{0}}^{2}=\left(\frac{c \alpha}{\Omega_{s} E}\right)^{2} \sigma_{\varepsilon_{0}}^{2}
$$

where $\mathcal{N}_{p}$ is the total number of photons emitted per electron per second at a dipole of bending radius $R, u_{p}$ is the photon energy, $\alpha$ is the momentum compaction factor, $\gamma$ is the normalized electron energy, $\Omega_{s}$ is the synchrotron oscillation angular frequency, and

$$
\mathcal{N}_{p}<u_{p}^{2}>=\frac{55 e^{2} c^{2} \hbar \gamma^{7}}{96 \sqrt{3} \pi \epsilon_{0} R^{3}}
$$

What is very important to realize is that the physical meaning of single particle "bunch length" $\sigma_{z_{0}}$ which describes the range of the uncertainty of the longitudinal position of the particle due to random quantum radiation excitations.

\section{BUNCH LENGTHENING AND ENERGY SPREAD INCREASING}

\subsection{Potential well distortion}

It is known that the mechanism for the potential well distortion which affects the bunch length, mainly due to the imaginary part of the impedance, is a static one, and the energy spread of the bunch is not affected [5]. It is useful to recall the corresponding general formulae from the potential well distortion theory, which are expressed as follows [2] [3]

$$
\begin{gathered}
\mathbf{R}_{z}^{2}=1+\frac{C_{P W D}^{\prime} I_{b}}{\mathbf{R}_{z}} \\
\mathbf{R}_{\varepsilon}=1
\end{gathered}
$$

The author of this paper, however, would believe that eq. 5 should be modified as follows:

$$
\mathbf{R}_{z}^{2}=1+\frac{C_{P W D} I_{b}}{\mathbf{R}_{z}^{1.5}}
$$

where $I_{b}$ is the bunch current, $\mathbf{R}_{z}=\sigma_{z} / \sigma_{z_{0}}$ and $\mathbf{R}_{\varepsilon}=$ $\sigma_{\varepsilon} / \sigma_{\varepsilon_{0}}$. The analytical expression of $C_{P W D}$ and the physical reason for the modification from eq. 5 to eq. 7 are given in ref. 8 . 


\subsection{Collective random excitations}

In this paper, we want to show that the resistive part of the impedance can lead to bunch energy spread increasing and bunch lengthening, and we will introduce this mechanism step by step.

- A bunch of length $\sigma_{z}$ with $N_{e}$ particles will lose a kinetic energy $W$ over one turn, and $W=e^{2} N_{e}^{2} \mathcal{K}_{\|}^{\text {tot }}\left(\sigma_{z}\right)$, where $\mathcal{K}_{\|}^{\text {tot }}\left(\sigma_{z}\right)$ is the longitudinal loss factor of the machine for one turn, which depends on the bunch length $\sigma_{z}$.

- The position of each particle inside the bunch is totally unpredictable. No matter where the particle is, after one synchrotron oscillation period $T_{s}$, each particle has experienced all the longitudinal positions in the bunch and each particle will lose in average an amount of energy $\mathcal{U}_{w}=e^{2} N_{e} \mathcal{K}_{\|}^{\text {tot }}\left(\sigma_{z}\right)$ per turn.

- The rf accelerating cavities in the ring will compensate $W$ for the bunch and $\mathcal{U}_{w}$ for each particle per turn in average.

- At the time $t=t_{i}$, a particle will find its longitudinal random position inside the bunch at $z_{i}$ which corresponds to a synchrotron phase $\phi_{i}$. Since the longitudinal wake potential varies with respect to the longitudinal position, a particle at one longitudinal position will lose an energy $\mathcal{U}_{i}$ after one turn.

- Since rf cavities provide each particle with an average energy gain $\mathcal{U}_{w}$ after each revolution, the net energy gain of the particle after one turn will be $\Delta E_{i}=\mathcal{U}_{w}-\mathcal{U}_{i}$. Apparently, if the particle is located at the very front of the bunch (denoted position 1) after one turn it will lose nothing due to the collective wake potential, however, it gets from rf cavities a gain of energy $\mathcal{U}_{w}$, and its corresponding $\Delta E_{1}=\mathcal{U}_{w}$. The second characteristic position for the particle in the bunch is $\Delta E_{2}=0$ which means the energy lost due to the wake potential is exactly compensated by the rf cavities. The third characteristic case is $\Delta E_{3}=-\mathcal{U}_{w}$ which corresponds to the longitudinal position where the particle feels the maximum wake potential. It is easy to know that $-\mathcal{U}_{w} \leq \Delta E_{i} \leq \mathcal{U}_{w}$. As stated above, after one synchrotron oscillation the average of $\Delta E_{i}$ over $T_{s}$ will be zero, and $\Delta E_{i}$ can be reasonably written as

$$
\Delta E_{i}=\mathcal{U}_{w} \sin \left(\Omega_{s} t-\phi_{i}\right)
$$

where $\phi_{i}$ is a random variable. The time interval to have $\Delta E_{i}$ and $\Delta E_{i+1}$ is the revolution period $T_{0}$. Eq. 8 decribes a stationary random collective excitation process on the energy of a particle in the bunch.

Including now the synchrotron damping effect, the effect of the random kick on the particle energy can be written as

$$
\Delta E_{i}=\mathcal{U}_{w} \exp \left(-\frac{t-t_{i}}{\tau_{\varepsilon}}\right) \sin \left(\Omega_{s}\left(t-t_{i}\right)\right)
$$

Having revealed the random process, we will calculate the rms value of the corresponding energy spread. This can be done by using Campbell's theorem [9] which gives

$$
\sigma_{\varepsilon, w}^{2}=\frac{\mathcal{U}_{w}^{2}}{4 T_{0}} \tau_{\varepsilon}
$$

Combining the effects from the quantum radiation and the collective random excitations (both random processes are statistically independent), one gets finally the resultant energy spread

$$
\begin{gathered}
\sigma_{\varepsilon}^{2}=\sigma_{\varepsilon_{0}}^{2}+\sigma_{\varepsilon, w}^{2} \\
=\sigma_{\varepsilon 0}^{2}\left(1+\frac{\mathcal{U}_{w}^{2}}{T_{0}<\mathcal{N}_{p}<u_{p}^{2}>>}\right)
\end{gathered}
$$

The bunch length connects with the bunch energy as follows

$$
\begin{gathered}
\sigma_{z}^{2}=\left(\frac{c \alpha}{\Omega_{s} E}\right)^{2} \sigma_{\varepsilon}^{2} \\
=\sigma_{z_{0}}^{2}\left(1+\frac{\mathcal{U}_{w}^{2}}{T_{0}<\mathcal{N}_{p}<u_{p}^{2}>>}\right)
\end{gathered}
$$

Defining $\mathbf{R}_{\iota}=\sigma_{\iota} / \sigma_{\iota 0}$, where $\iota$ represents either the bunch length or energy spread, for an isomagnetic ring one gets

$$
\mathbf{R}_{\iota}^{2}=\left(1+\frac{144 \epsilon_{0}\left(e R N_{e} \mathcal{K}_{\|}^{\text {tot }}\left(\sigma_{z}\right)^{2}\right.}{55 \sqrt{3} \hbar c \gamma^{7}}\right)
$$

The dependence of $\mathcal{K}_{\|}^{\text {tot }}$ on the bunch length can be expressed as

$$
\mathcal{K}_{\|}^{\text {tot }}=\mathcal{K}_{\|, 0}^{\text {tot }}\left(\frac{\sigma_{z_{0}}}{\sigma_{z}}\right)^{\varsigma}
$$

where $\mathcal{K}_{\|, 0}^{\text {tot }}$ is the longitudinal loss factor at $\sigma_{z}=\sigma_{z_{0}}$, and $\sigma_{z_{0}}$ is the natural bunch length. If SPEAR scaling law [10] is used (for example), $\varsigma \approx 1.21$ (each machine has its own $\varsigma$ ), and eq. 13 can be written as

$$
\mathbf{R}_{\iota}^{2}=1+\frac{\mathcal{C}\left(R_{a v} R I_{b} \mathcal{K}_{\|, 0}^{\text {tot }}\right)^{2}}{\gamma^{7} \mathbf{R}_{\iota}^{2.42}}
$$

where

$$
\mathcal{C}=\frac{576 \pi^{2} \epsilon_{0}}{55 \sqrt{3} \hbar c^{3}}
$$

$I_{b}=e N_{e} c / 2 \pi R_{a v}$ and $R_{a v}$ is the average radius of the ring.

Now it is time for us to combine the effects from both potential well distortion and collective random excitations. Including eqs. 7 and 6 into eq. 15, eq. $15(\iota=z)$ becomes

$$
\mathbf{R}_{z}^{2}=1+\frac{C_{P W D} I_{b}}{\mathbf{R}_{z}^{1.5}}+\frac{\mathcal{C}\left(R_{a v} R I_{b} \mathcal{K}_{\|,, 0}^{\text {tot }}\right)^{2}}{\gamma^{7} \mathbf{R}_{z}^{2.42}}
$$

and eq. $15(\iota=\varepsilon)$ remains

$$
\mathbf{R}_{\varepsilon}^{2}=1+\frac{\mathcal{C}\left(R_{a v} R I_{b} \mathcal{K}_{\|, 0}^{\mathrm{tot}}\right)^{2}}{\gamma^{7} \mathbf{R}_{z}^{2.42}}
$$

What should be pointed out is that $\mathcal{C}$ is a positive number, however, $C_{P W D}$ can be negative if the momentum compaction factor, $\alpha$, is negative. The procedure to get the information about the bunch lengthening and the energy spread increasing is firstly to solve eq. 17 and find $\mathbf{R}_{z}\left(I_{b}\right)$, and then calculate $\mathbf{R}_{\varepsilon}\left(I_{b}\right)$ by putting $\mathbf{R}_{z}\left(I_{b}\right)$ into eq. 18 . 
When the bunch current is high enough to neglect the effect of PWD, one has $\mathbf{R}_{\varepsilon} \approx \mathbf{R}_{z}$ which means that energy spread increasing and bunch lengthening are almost correlated.

To finish this section, we point out that the third term in eq. 17 might correspond to the so-called turbulent bunch lengthening observed in the experiments.

\section{COMPARISON WITH EXPERIMENTAL RESULTS}

In this section we use the theory developed above to explain the experimental bunch lengthening measurement results in the storage rings of BEPC and Super-ACO. In the following, we shall fit the experimental bunch lengthening curves by eq. 17 and extract the information on the total loss factor at the natural bunch length for the corresponding machines.

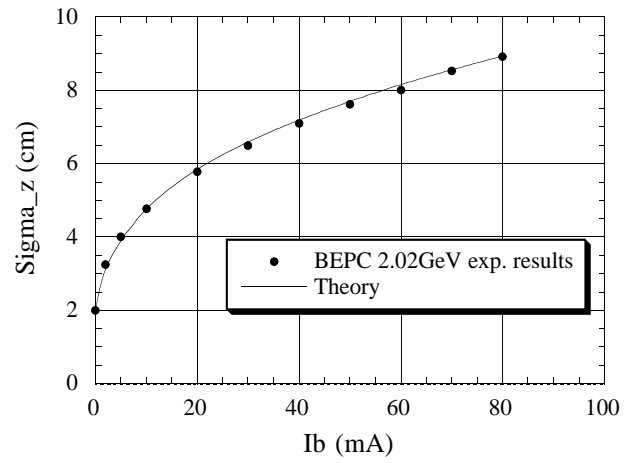

Figure 1: Comparison between BEPC $(R=10.345 \mathrm{~m}$ and $R_{a v}=38.2 \mathrm{~m}$ ) experimental results and the theoretical results at $2.02 \mathrm{GeV}$ with $\sigma_{z_{0}}=2 \mathrm{~cm}$. The continuous line is the fitted curve of the theory. The dark points are the BEPC $2.02 \mathrm{GeV}$ experimental results.

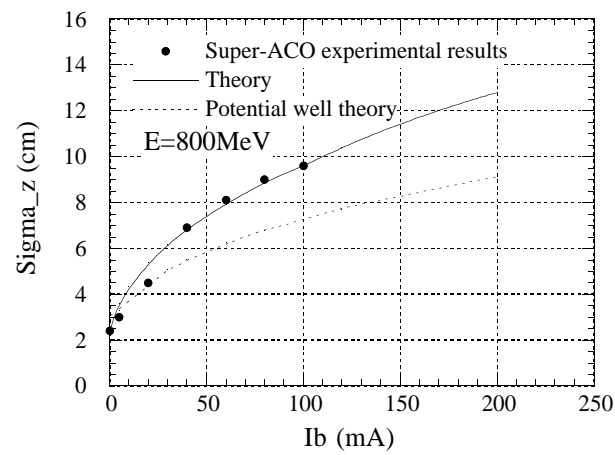

Figure 2: Comparison between Super-ACO $(R=1.7 \mathrm{~m}$ and $R_{a v}=11.5 \mathrm{~m}$ ) experimental results, the theoretical results and the potential well distortion theory predictions at $800 \mathrm{MeV}$ with $\sigma_{z_{0}}=2.4 \mathrm{~cm}$. The continuous and the dotted lines are the fitted curves of the new and the potential well distortion theories, respectively. The dark points are the Super-ACO $800 \mathrm{MeV}$ experimental results.

Firstly, we look at BEPC (Beijing Electron Positron Collider) [11]. Fig. 1 shows the comparison results between the new theory and the experimental results [11]. By fitting one finds $\mathcal{K}_{\|, 0}^{\text {tot }}=3.82 \mathrm{~V} / \mathrm{pC}$ at $\sigma_{z_{0}}=2 \mathrm{~cm}$ which corresponds $\mathcal{K}_{\|}^{\text {tot }}=1.65 \mathrm{~V} / \mathrm{pC}$ at $\sigma_{z}=4 \mathrm{~cm}$ by using SPEAR scaling. The recent experimental result [12] shows that $\mathcal{K}_{\|}^{\text {tot }}=1.5 \mathrm{~V} / \mathrm{pC}$ at $\sigma_{z}=4 \mathrm{~cm}$ which agrees quite well with the theoretical result.

Secondly, Super-ACO [13] is considered and the comparison results are shown in Fig. 2. From the experimental curve one finds $\mathcal{K}_{\|, 0}^{\text {tot }}=3.6 \mathrm{~V} / \mathrm{pC}$ at $\sigma_{z_{0}}=2.4 \mathrm{~cm}$. The total loss factor of the machine at $\sigma_{z}=3 \mathrm{~cm}$ is calculated as $2.22 \mathrm{~V} / \mathrm{pC}$ by using TBCI [14], and the $\mathcal{K}_{\|}^{\text {tot }}$ obtained from the experimental bunch lengthening curve is $2.76 \mathrm{~V} / \mathrm{pC}$ for the same bunch length by using SPEAR scaling. What should be mentioned is that the experimental results shown in Fig. 2 correspond to the case of positive momentum compaction factor $\alpha=0.0148$.

More comparisons results can be found in ref. 15 .

\section{CONCLUSION}

In this paper a bunch lengthening theory is established and it is demonstrated that the longitudinal resistive part impedance of the machine (loss factor) increase the bunch length and the energy spread due to collective random excitation effects. Some comparisons between experimental and theoretical results are made and the agreements are quite well.

\section{ACKNOWLEDGEMENTS}

I would like to thank J. Le Duff for useful discussions and critical comments.

\section{REFERENCES}

[1] R. Belbeoch et al., Proceedings of the national conference on particle accelerators, No. 1 (Moscow, 1968) p. 129 (in Russian).

[2] C. Pellegrini and A.M. Sessler, Nuovo Cimento, 3A (1971) p. 116.

[3] J. Haissinski, Nuovo Cimento, 18B (1973) p. 72.

[4] D. Boussard, CERN LabII/RF/int./75-2 (1975).

[5] A.W. Chao, "Physics of collective beam instabilities in high energy accelerators", Wiley, New York, 1993.

[6] J. Gareyte, Advances of accelerator physics and technologies, (Editor H. Schopper), World Scientific, 1993, p. 67.

[7] M. Sands, SLAC-121 (1970).

[8] J. Gao, personal note to be published.

[9] J.J. Freeman, "Principles of noise", John Wiely \& Sons, Inc. p. 99.

[10] P.B. Wilson, et al., IEEE Trans. on Nucl. Sci. Vol. NS-24, No. 3, June 1977, p. 1211.

[11] Z. Guo, et al., Proceedings of PAC95, Dallas TX (1995) p. 2955.

[12] M. Tigner, Private communication, 03/04/1997.

[13] A. Nadji, et al., Proceedings of EPAC96, Barcelona (1996) p. 676.

[14] P. Brunelle, thèse, 1990.

[15] J. Gao, LAL/RT 97-01, February 1997. 